\title{
COVID-19 Crisis Effects on Caregiver Distress in Neurocognitive Disorder
}

Panagiotis Alexopoulos ${ }^{\mathrm{a}, \mathrm{b}, \mathrm{c}, \mathrm{d}, *}$, Rigas Soldatos $^{\mathrm{e}}$, Evangelia Kontogianni $^{\mathrm{e}}$, Maria Frouda ${ }^{\mathrm{d}}$, Souzana loanna Aligianni ${ }^{\mathrm{a}}$, Maria Skondra ${ }^{\mathrm{a}, \mathrm{c}, \mathrm{f}}$, Maria Passa ${ }^{\mathrm{e}}$, Georgia Konstantopoulou ${ }^{\mathrm{g}}$, Evangelia Stamouli ${ }^{\mathrm{e}}$ Evgenia Katirtzoglou ${ }^{\mathrm{e}}$, Anastasios Politis ${ }^{\mathrm{h}}$, Polychronis Economou ${ }^{\mathrm{i}}$, Maria Alexaki ${ }^{\mathrm{e}}$, Kostas Siarkos ${ }^{\mathrm{e}}$ and Antonios Politis ${ }^{\mathrm{e}, \mathrm{j}}$

${ }^{a}$ Department of Psychiatry, Patras University Hospital, Faculty of Medicine, School of Health Sciences, University of Patras, Patras, Greece

${ }^{\mathrm{b}}$ Department of Psychiatry and Psychotherapy, Klinikum rechts der Isar, Faculty of Medicine, Technische Universität München, Munich, Germany

${ }^{\mathrm{c}}$ Psychogeriatric unit for neurocognitive assessment and caregiver counselling, Patras Office of The Hellenic Red Cross, Patras, Greece

${ }^{\mathrm{d}}$ Patras Dementia Day Care Center, Corporation for Succor and Care of Elderly and Disabled - FRODIZO, Patras, Greece

${ }^{\mathrm{e}}$ First Department of Psychiatry, Eginition Hospital, School of Medicine, National and Kapodistrian University of Athens, Athens, Greece

${ }_{\mathrm{f}}^{\mathrm{f}}$ Department of Nursing, School of Health Rehabilitation Sciences, University of Patras, Patras, Greece ${ }^{\mathrm{g}}$ Special Office for Health Consulting Services and Faculty of Education and Social Work, School of Humanities and Social Sciences, University of Patras, University of Patras, Patras, Greece

${ }^{\mathrm{h}}$ Charing Cross Hospital, Department of Neurosurgery, Imperial College London

${ }^{\mathrm{i}}$ Department of Civil Engineering (Statistics), University of Patras, Patras, Greece

${ }^{\mathrm{j}}$ Department of Psychiatry, Division of Geriatric Psychiatry and Neuropsychiatry, Johns Hopkins Medical School, Baltimore, MD, USA

Accepted 3 November 2020

\begin{abstract}
.
Background: The outbreak of the COVID-19 pandemic seems to have mental health implications for both people with neurocognitive disorder and their caregivers.

Objective: The study aimed to shed light on relations between caregiver mental reaction to the pandemic and caregiver distress related to neuropsychiatric symptoms, memory impairment progression, and functional impairment of people with neurocognitive disorder during the period of confinement in Greece.

Methods: The study included caregivers of patients with mild $(\mathrm{N}=13)$ and major $(\mathrm{N}=54)$ neurocognitive disorder. The caregiver-based telephone interview was based on items of the neuropsychiatric inventory questionnaire, the AD8 Dementia Screening Instrument, and the Bristol Activities of Daily Living Scale. Regarding the mental impact of the COVID-19 crisis on caregivers, four single questions referring to their worries in the last seven days were posed, in addition to the scales Generalized Anxiety Disorder 7-Item (GAD-7) and the 22-item Impact of Event Scale-revised (IES-R). A stepwise linear regression model was employed for studying the relationship between caregiver distress and demographic and clinical data and caregiver mental reaction to COVID-19 pandemic outbreak.
\end{abstract}

${ }^{*}$ Correspondence to: Dr. Panagiotis Alexopoulos, Department of Psychiatry, University Hospital of Rion, 26504 Rio, Patras,
Greece. Tel.: +302613603728; Fax: +302610994534; E-mail: panos.alexopoulos@upatras.gr. 
Results: Caregiver distress severity during the confinement period was influenced not only by memory deficits $(p=0.009)$ and neuropsychiatric symptoms $(p<0.001)$ of patients, but also by caregiver hyperarousal $(p=0.003)$ and avoidance symptoms $(p=0.033)$ and worries directly linked to the COVID-19 crisis $(p=0.022)$.

Conclusion: These observations provide further evidence for the urgent need for support of caregivers of patients with neurocognitive disorder during the COVID-19 pandemic.

Keywords: Confinement, COVID-19, impairment in activities of daily living, memory deficits, neuropsychiatric symptoms

\section{INTRODUCTION}

Caring for people with neurocognitive disorder is accompanied by distress for the caregiver. The terms major and mild neurocognitive disorder have been proposed by the fifth edition of the Diagnostic and Statistical Manual of Mental Disorders (DSM-5) and are equivalent to the terms dementia and mild cognitive impairment, respectively [1]. They manifest with a triad of symptom groups: 1) cognitive deficits (e.g., memory), 2) neuropsychiatric symptoms such as anxiety, and 3) decline in activities of daily living (ADL) [2]. Even people with mild neurocognitive disorder suffer from mild decline in instrumental ADL (e.g., transportation, managing finances) [3]. Individuals with neurocognitive disorder need comprehensive support depending on the severity of their symptoms [3]. Most of them are supported at home by informal caregivers (e.g., spouses, children), a duty or responsibility which is not rarely experienced as hard to bear [4]. Mounting evidence points to the impact of neuropsychiatric symptoms and the severity of cognitive and functional impairment on caregiver distress, negatively affecting both caregiver mental health and patients' care [4-8].

The recent outbreak of the SARS-CoV-2 (COVID19) pandemic has implications for the mental health of individuals with neurocognitive disorder and their caregivers as well as for the general population [9]. Draconic measures such as social distancing, confinement, and quarantine aiming to contain the COVID-19 spread, in combination with the fear and the uncertainty that are caused by the lack of vaccines and effective medical treatments, were shown to pertain to psychological distress, anxiety, and mood disturbances or even to symptoms of posttraumatic stress disorder, since the pandemic outbreak of infections, the outcome of which is hardly predictable, can be perceived as a traumatic experience [9-11]. In Greece during confinement (March 23 to May 4,2020 ), people were only permitted to leave their homes to buy essential good, briefly exercise or seek medical help, or, in specific cases, go to work (e.g., health workforce). Interestingly, recent reports unraveled that confinement was related to worsening of cognitive and neuropsychiatric symptoms, to functional decline, and increase in caregiver burden [12-14].

The current study provides further pieces of evidence on the effects of the COVID-19 crisis on caregiver distress. It aimed to shed light on the potential relations between caregiver mental reaction to the pandemic and caregiver distress related to neuropsychiatric symptoms, memory impairment progression, and performance in ADL of people with neurocognitive disorder during the period of confinement in Greece. In such a way, the urgent need for the development of pragmatic strategies to support caregivers in the new terrain of the COVID-19 pandemic can become evident.

\section{METHODS}

\section{Participants}

During the confinement, 67 caregivers of people with mild or major neurocognitive disorder who had attended the psychogeriatrics outpatient clinic of the Eginition Hospital in Athens, the outpatient unit of the dementia day care center of the Corporation for Succor and Care of Elderly and Disabled - FRODIZO in Patras, or the psychogeriatric unit for neurocognitive assessment and caregiver counselling of the local office of the Hellenic Red Cross, were asked over the phone to participate in the study. Inclusion criteria were: 1) being the main informal caregiver of a patient with neurocognitive disorder and 2) verbal consent for a telephone interview prior to study enrolment. Exclusion criteria included: 1) current acute/unstable phase of a mental disorder of the caregiver, 2) COVID-19 infection or flu-like self-limiting symptoms of the caregiver or his/her family members or close relatives/friends during the confinement, 3) active involvement as medical or non-medical professional in the management of the COVID-19 pandemic, or 4) being a caregiver of an individual living in assisted living facilities or nursing care 
facilities. The diagnosis of mild and major neurocognitive disorder was based on the DSM-5 diagnostic criteria [15]. The diagnoses of Alzheimer's disease [16], cerebrovascular disease [17], frontotemporal lobar degeneration [18], or neurocognitive disorder due to mixed etiology $[19,20]$ had been established according to international diagnostic criteria by a psychiatrist or neurologist after an extensive diagnostic workup in the six months preceding the introduction of the restriction measures. The study was conducted in accordance with the latest revision of the Declaration of Helsinki and was approved by the ethics committee of the Kapodistrian and National University of Athens.

\section{Procedures}

A caregiver-based telephone interview format was employed. A standardized set of questions, regarding both their distress and mental reaction to COVID19 crisis, as well as patients' symptoms, was asked to caregivers during the confinement (Table 1). To assess the development of memory deficits, neuropsychiatric symptoms, functional impairment, and the caregiver distress pertaining to them, Likert items were employed. Neuropsychiatric symptoms were assessed with the neuropsychiatric inventory questionnaire [21], while items assessing memory impairment progression and functional impairment were based on the AD8 Dementia Screening Instrument [22] and the Bristol Activities of Daily Living Scale [23]. A distress composite score was calculated based on the sum of caregiver distress due to memory impairment progression, due to neuropsychiatric symptoms, and due to functional impairment. Except for the assessment of distress related to neuropsychiatric symptoms, the items of which are measured on a six-point Likert scale (0-5, with 0 meaning not distressing at all and 5 meaning extremely distressing), the number of options of the Likert items on which the assessment of distress linked to memory and functional impairment was based, was four (0-3, with 0 meaning not distressing at all and 3 meaning extremely distressing). Considering the distribution of caregivers' responses to the NPI distress items, the Likert items 3, 4, and 5 were classified together, so that all component contributions are codified according to the same schema. Regarding the mental impact of the COVID-19 crisis on caregivers, four single questions referring to their worries in the last seven days were posed. The caregiver responses to them were summed up in a composite score (Table 1).
In addition, the well-established scales Generalized Anxiety Disorder 7-Item (GAD-7) [24] and the 22item Impact of Event Scale-revised (IES-R) [25] were employed. The IES-R assesses avoidance (the tendency to avoid thoughts or reminders of the pandemic), intrusion (difficulties in staying asleep, dissociative experiences similar to flashbacks) and hyperarousal (irritated feeling, difficulties in sleep onset) [25].

Of note, data on neuropsychiatric symptoms during the pre-lockdown period was available for patients of the Eginition Hospital who were included in the present study. In particular, for 34 patients data on neuropsychiatric symptoms and the caregiver distress related to them from the last assessment of these 34 patient and caregiver dyads in the two months preceding the introduction of the COVID-19 restrictive measures was available and was analyzed to grasp alterations in these symptoms between the pre-lockdown period and the confinement.

\section{Statistical analyses}

Cronbach's alpha was used as a measure of internal consistency of the questionnaire's items employed to assess COVID-19 related worries (four items), impairment in ADL (three items), and distress related to it (three items). The assessment of the reliability of the Cronbach's alpha coefficients was based on the value of the first eigenvalue of the Principal Component Analysis for the items considered to obtain the Cronbach's alpha [26]. Differences in neuropsychiatric symptoms and distress related to them in the subsample with available data for both the time periods prior and during confinement were studied with the Wilcoxon test, since data normality assumption was rejected based on analysis of skewness and kurtosis. Stepwise linear regression model (alpha to enter $=0.15$, alpha to remove $=0.15$ ) was employed for studying the relationship between caregiver distress and demographic and clinical data and caregiver mental reaction to COVID-19 pandemic outbreak.

\section{RESULTS}

The study sample included 67 caregivers of 45 patients with $\mathrm{AD}$, of nine patients with brain vascular disease, of two patients with frontotemporal lobar degeneration and of eleven patients with neurocognitive disorder due to mixed etiology (Table 2). The internal consistency of the items which were employed to assess COVID-19 related worries, func- 
Table 1

Rating tools employed to assess symptoms of neurocognitive disorders and the distress related to them

\begin{tabular}{|c|c|c|}
\hline Symptoms (in the last seven days) & $\begin{array}{l}\text { Symptom rating scale } \\
\text { (Number of options of } \\
\text { the Likert items) }\end{array}$ & $\begin{array}{l}\text { Caregivers' burden } \\
\text { assessment (Number of } \\
\text { options of the } \\
\text { Likert items) }\end{array}$ \\
\hline Cognitive function & $($ Severity)* & (Severity)* \\
\hline Memory deficits progression & $0-3$ & $0-3$ \\
\hline Neuropsychiatric symptoms & $(\text { Frequency })^{\dagger} \times(\text { Severity })^{* *}$ & $(\text { Severity })^{* * *}$ \\
\hline Delusions & $(0-4) \times(1-3)$ & $0-5$ \\
\hline Hallucinations & $(0-4) \times(1-3)$ & $0-5$ \\
\hline Agitation/Aggression & $(0-4) \times(1-3)$ & $0-5$ \\
\hline Depression & $(0-4) \times(1-3)$ & $0-5$ \\
\hline Anxiety & $(0-4) \times(1-3)$ & $0-5$ \\
\hline Elation/Euphoria & $(0-4) \times(1-3)$ & $0-5$ \\
\hline Apathy/Indifference & $(0-4) \times(1-3)$ & $0-5$ \\
\hline Disinhibition & $(0-4) \times(1-3)$ & $0-5$ \\
\hline Irritability & $(0-4) \times(1-3)$ & $0-5$ \\
\hline Aberrant motor behaviors & $(0-4) \times(1-3)$ & $0-5$ \\
\hline Sleep and night time behavior disorders & $(0-4) \times(1-3)$ & $0-5$ \\
\hline Appetite and eating disorders & $(0-4) \times(1-3)$ & $0-5$ \\
\hline Activities of daily living (ADL) & $($ Severity)* & (Severity)* \\
\hline Difficulties with personal hygiene & $0-3$ & \\
\hline Difficulties with self-care & $0-3$ & \\
\hline Difficulties with physical health protection / management & $0-3$ & $0-3$ \\
\hline $\begin{array}{l}\text { Difficulties with participation in social activities (e.g., } \\
\text { leisure activities, physician office visit) }\end{array}$ & & $0-3$ \\
\hline Difficulties with communication & & $0-3$ \\
\hline COVID 19 related worries that & $(\text { Severity })^{*}$ & \\
\hline (i) the patient will get infected by COVID 19 & $0-3$ & \\
\hline (ii) the patient will succumb to death because of COVID 19 & $0-3$ & \\
\hline (iii) the patient will be infected by the caregiver & $0-3$ & \\
\hline $\begin{array}{l}\text { (iv) that something bad will happen to the caregiver } \\
\text { and his/her family }\end{array}$ & $0-3$ & \\
\hline Generalized Anxiety Disorder 7-Item & (Frequency) ${ }^{\dagger \dagger} 0-3 \times 7$ items & \\
\hline Impact of event scale-revised (IES-R) & $(\text { Severity })^{\dagger \dagger \dagger}$ & \\
\hline Intrusion & $0-4 \times 8$ items & \\
\hline Avoidance & $0-4 \times 8$ items & \\
\hline Hyperarousal & $0-4 \times 6$ items & \\
\hline
\end{tabular}

${ }^{*} 0=$ Not at all, $1=$ A little bit, $2=$ Moderately, $3=$ Severely. ${ }^{* *} 1=$ Mild (noticeable, but not a significant change), $2=$ Moderate (significant, but not a dramatic change), $3=$ Severe (very marked or prominent, a dramatic change). ${ }^{* * *} 0=$ Not distressing at all, $1=$ Minimal (slightly distressing, not a problem to cope with), $2=$ Mild (not very distressing, generally easy to cope with), $3=$ Moderate (fairly distressing, not always easy to cope with), $4=$ Severe (very distressing, difficult to cope with), $5=$ Extreme or very Severe (extremely distressing, unable to cope with). ${ }^{\dagger} 0=$ Never, $1=$ Occasionally (less than once per week), $2=$ Often about once per week), $3=$ Frequently (several times per week but less than every day), $4=$ Very frequently (daily or essentially continuously present). ${ }^{\dagger \dagger} 0=$ Not at all, $1=$ Several days, $2=$ More than half the days, $3=$ Nearly every day. ${ }^{\dagger \dagger} 0=$ Not at all, $1=$ A little bit, $2=$ Moderately, $3=$ Quite a bit, $4=$ Extremely.

tional impairment, and distress related to it was good to excellent (Cronbach alpha correlation coefficients were $0.87,0.93$, and 0.64 , respectively). Of note, based on a previous simulation study [26], the estimated simulated relative bias for a sample size of 67 is approximately equal to -0.05 and -0.015 , whenever the first eigenvalue is smaller than 3 and between 3 and 6 , respectively. In the present study, the first eigenvalues for each case were $2.87,2.62$, and 1.74 indicating that the relative bias of the Cronbach's alpha is not expected to be any larger than -0.05 . Neuropsychiatric symptoms did not significantly vary between the assessment prior the confinement and that during it $(Z=-1.56, p=0.12)$. In contrast, change in emotional distress related to these symptoms tended to be significant $(Z=-1.82, p=0.07)$. The stepwise regression included the distress composite score as dependent variable and demographic data and clinical symptoms that are included in Table 2 and refer to the confinement as independent variables (18 variables in total). The final selected model (Table 3) revealed a significant impact of neuropsychiatric symptoms, hyperarousal, avoidance, memory deficits progression, and worries related to COVID-19, while the city at which participants lived, included in the analysis as a dichotomic variable, tended to exert a 
Table 2

Characteristics of the study sample

\begin{tabular}{|c|c|}
\hline $\mathrm{N}$ & 67 \\
\hline Recruited in Athens & 34 \\
\hline Recruited in Patras & 33 \\
\hline Caregivers' age $\neq$ & $58.31(12.20)[32-80]$ \\
\hline Caregivers' sex (female, $\mathrm{N}$ ) & 55 \\
\hline Caregivers' education $(\mathrm{y})^{\neq}$ & $14.07(3.96)[6-23]$ \\
\hline \multicolumn{2}{|l|}{ Caregiver's relationship to patient $(\mathrm{N})$} \\
\hline Spouse & 20 \\
\hline Children & 43 \\
\hline Brother/sister & 1 \\
\hline Other & 3 \\
\hline Duration of involvement in patient's care per day (in $\mathrm{h})^{\neq}$ & $7.50(6.51)[0.01-16.00]$ \\
\hline \multicolumn{2}{|l|}{ Patients with different severity degree of neurocognitive disorder (N) } \\
\hline Mild neurocognitive disorder $(\mathrm{N})$ & 13 \\
\hline Mild major neurocognitive disorder $(\mathrm{N})$ & 19 \\
\hline Moderate major neurocognitive disorder $(\mathrm{N})$ & 29 \\
\hline Severe major neurocognitive disorder $(\mathrm{N})$ & 6 \\
\hline Patients' age $\neq$ & $80.21(7.70)[63-92]$ \\
\hline Patients' sex (female, $\mathrm{N}$ ) & 47 \\
\hline Memory deficits progression $\neq$ & $0.85(0.96)[0-23]$ \\
\hline Neuropsychiatric symptoms $\neq$ & $18.31(19.01)[0-70]$ \\
\hline Impairment in activities of daily living (functional impairment) $\neq$ & $3.96(1.99)[0-9]$ \\
\hline Confinement length at the assessment time point (in days) ${ }^{\neq}$ & $31.99(6.86)[17-47]$ \\
\hline COVID-19 related worries $\neq$ & $4.58(3.73)[0-12]$ \\
\hline General Anxiety Disorder 7-Item $\neq$ & $5.18(4.90)[0-20]$ \\
\hline \multicolumn{2}{|l|}{ Impact of event scale-revised (IES-R) } \\
\hline Intrusion $\neq$ & $5.64(7.03)[0-27]$ \\
\hline Avoidance $\neq$ & $4.91(5.61)[0-22]$ \\
\hline Hyperarousal $\neq$ & $5.88(5.09)[0-23]$ \\
\hline Caregivers' distress during confinement (composite score) ${ }^{\neq}$ & $10.37(8.50)[0-31]$ \\
\hline Distress related to memory deficits $\neq$ & $1.03(1.03)[0-3]$ \\
\hline Distress related to neuropsychiatric symptoms ${ }^{\neq}$ & $7.37(6.54)[0-23]$ \\
\hline Distress related to functional impairment $\neq$ & $0.76(0.89)[0-3]$ \\
\hline \multicolumn{2}{|l|}{ Prior confinement } \\
\hline Neuropsychiatric symptoms $\not$ & $(\mathrm{N}=34), 9.79(9.94)[0-38]$ \\
\hline Caregivers' distress related to neuropsychiatric symptoms $\neq$ & $(\mathrm{N}=34), 6.00(4.68)[0-16]$ \\
\hline
\end{tabular}

significant influence. In particular, the difference in the expected value of the distress composite scores between two caregivers with the same characteristics is 2.16 degrees with the caregiver living in Athens (reference city) manifesting a higher score.

\section{DISCUSSION}

Casting light on the impact of the COVID-19 outbreak on caregiver distress is of paramount importance, since caregivers are called to manage the multifaceted symptoms and needs of patients despite they have only limited external support [13]. The present study, which was based on an in-depth assessment of the caregiver mental reaction to the COVID-19 pandemic, included caregivers of people in different stages of neurocognitive disorder and subsequently different care needs, whilst a wide spectrum of symptoms of neurocognitive disorder was considered. According to our findings caregiver distress linked to the triad of symptom groups of neurocognitive disorder was significantly influenced by caregiver worries regarding the risk of contagion and death, hyperarousal symptoms (e.g., irritability, hypervigilance) and avoidance behavior (e.g., denial of the meanings and consequences of the pandemic) [25]. In line with the unveiled association between caregiver distress and their mental 
Table 3

Impact of neuropsychiatric symptoms and caregivers' mental reaction to COVID-19 crisis on caregiver distress related to symptoms of neurocognitive disorder according to the final selected regression model

\begin{tabular}{ll}
\hline Stepwise Regression model & adjusted $\mathrm{R}^{2}=0.83$ \\
Caregivers' distress during confinement (composite score) & $\mathrm{F}=53.32, p<0.001$ \\
Independent Variables & Coefficient $(p$-value) \\
Hyperarousal & $0.356, p=0.003^{* *}$ \\
Avoidance & $-0.2180, p=0.033^{*}$ \\
Worries related to COVID-19 & $0.326, p=0.022^{*}$ \\
Neuropsychiatric symptoms & $0.3701, p<0.001^{* * *}$ \\
Memory deficits progression & $1.324, p=0.009^{* *}$ \\
City (place of residence, Athens reference city) (dichotomic variable) & $-2.16, p=0.057$ \\
Constant & $3.18, p=0.042^{*}$ \\
\hline
\end{tabular}

*significant at 0.05 level, ${ }^{* *}$ significant at 0.01 level, ${ }^{* * *}$ significant at 0.001 level.

reaction to COVID-19 crisis is the observation that there is a trend to significance in the increase in distress linked to neuropsychiatric symptoms between the time period preceding the pandemic outbreak and during the confinement, despite the absence of significant alterations in neuropsychiatric symptoms between the two time points in the subsample with available data. Our observation regarding changes between the two time points may not apply to the entire group, since the subsample with available data for both time points differed from the rest of patient and caregiver dyads in diagnoses' distribution (Pearson Chi square, $p=0.001$ ), caregivers' sex distribution (Fischer's exact test, $p=0.023$ ), and in the age of the patients (Mann-Whitney test, $p=0.04$ ). Of note, the relationship between distress and avoidance, as assessed with the valid IES-R, was inverse. This finding warrants further investigation since a positive correlation would have been expected. Nonetheless, it may mirror the protective effects of avoidance behaviors which, may embody a way to cope with the negative impact of the COVID-19 related dramatic changes in people's life, which may not fundamentally constrain quality of life in the exceptional circumstances of the lockdown. The lack of significant association between the IES-R intrusion score and distress could also be a topic of future research. It may pertain to the unique characteristics of the COVID-19 pandemic as trauma [9]. Generalized anxiety symptoms and worries assessed with the General Anxiety Disorder 7-Item instrument were not found to be significantly correlated with caregivers' distress pertaining to memory impairment neuropsychiatric symptoms and functional impairment. In such a way, it becomes evident that the anxiety symptoms and worries linked to caregivers' distress do not pursue a generalized character but are directly related to the COVID-19 pandemic [27]. Thus, it can be reckoned that COVID-19 mental reaction influences even dis- tress feelings which pertain to caregivers' difficulties with symptom management.

Only neuropsychiatric symptoms and memory deficits but not functional impairment were shown to be related to caregivers' distress linked to neurocognitive disorders. In line with previous observations, caregivers felt more distressed if cognitive deficits and neuropsychiatric symptoms were more severe and patients subsequently needed more intensive supervision and support [5, 12]. Contrary to past reports, functional impairment and subsequently degree of severity of neurocognitive disorder were not found to be related to caregivers' distress [4-8]. Despite being somehow unexpected, this observation can be easily interpreted in the light of the circumstances in which the present study was conducted. Confinement probably enabled caregivers to have more time for caring for persons with neurocognitive disorders, while the lockdown constellation may have minimized the impact of functional impairment, since patients and their caregivers were confined to their homes and caregivers had more time for providing care. The revealed trend toward statistical significance for the association between living in Athens versus Patras and caregiver distress should be treated with caution. It may mirror differences in stress perception during confinement between inhabitants of a global city (Athens) and a regiopolis (Patras) [28].

The present study has a number of limitations. The representative nature is obviously limited because of the small sample size and the study temporal restriction to the period of confinement. Moreover, owing to the cross-sectional study design, no conclusions with regard to causality can be drawn. The assessment of functional impairment and memory deficits as well as of caregiver distress related to them were not based on validated instruments (e.g., on a telephone-based cognitive test), but relied on caregivers' reports on patients' memory deficits progression and functional 
ability and on questions directly grasping caregiver difficulties with the management of memory deficits and functional impairment. The internal consistency of the employed items was good. This strategy was a reasonable compromise between very long interviews over the phone and a pragmatic assessment of caregiver distress related to the whole triad of symptom groups of neurocognitive disorder. In addition, non-amnestic cognitive deficits (e.g., executive dysfunction), medical comorbidities, therapy adjustments, caregiver subjective sources of burden such as personality structure, and current life situation [4-7] were not considered in the present study, despite their influence on caregiver distress.

Further studies are warranted before final conclusions with regard to the relationship between the mental reaction to COVID-19 pandemic and distress related to memory deficits, neuropsychiatric symptoms, and functional impairment of people with neurocognitive disorder can be drawn. The observations of the present study clearly highlight the significant effects of caregiver hyperarousal and avoidance symptoms and worries directly linked to COVID-19 crisis on their distress. They serve as an alarming voice for the support needs of caregivers of people with neurocognitive disorder during the COVID-19 pandemic. In the light of reduction or even suspension of services dedicated to the management of neurocognitive disorder (e.g., day centers) during the pandemic, our findings provide evidence for the necessity to support carers, in order to keep them from burning out and experiencing health decline [29]. Web- or telephone-based interventions to foster social engagement and facilitate physical activity embody realistic strategies, in addition to mental health support hotlines [30]. In the long run, designing and implementing community outreach projects providing (online) peer support and building intergenerational mentoring networks based on telephone and online contacts, as well as improving conventional ways of providing home care to elderly people at small teams (e.g., Buurtzorg home care model) [31] may form ways to alleviate caregiver distress in the era of social disconnection due to the COVID-19 pandemic.

\section{ACKNOWLEDGMENTS}

This research received no specific grant from any funding agency, commercial or not-for-profit sectors.

Authors' disclosures available online (https:// www.j-alz.com/manuscript-disclosures/20-0991r3).

\section{REFERENCES}

[1] Stokin GB, Krell-Roesch J, Petersen RC, Geda YE (2015) Mild neurocognitive disorder: an old wine in a new bottle. Harv Rev Psychiatry 23, 368-376.

[2] Politis AM, Alexopoulos P, Vorvolakos T (2020) May neuropsychiatric symptoms be a potential intervention target to delay functional impairment in Alzheimer's disease? Int Psychogeriatr 32, 689-691.

[3] Perneczky R, Pohl C, Sorg C, Hartmann J, Komossa K, Alexopoulos P, Wagenpfeil S, Kurz A (2006) Complex activities of daily living in mild cognitive impairment: conceptual and diagnostic issues. Age Ageing 35, 240-245.

[4] Seidel D, Thyrian JR (2019) Burden of caring for people with dementia - comparing family caregivers and professional caregivers. A descriptive study. J Multidiscip Healthc 12, 655-663.

[5] Schumann C, Alexopoulos P, Perneczky R (2019) Determinants of self- and carer-rated quality of life and caregiver burden in Alzheimer disease. Int J Geriatr Psychiatry 34, 1378-1385.

[6] Kang HS, Myung W, Na DL, Kim SY, Lee J-H, Han S-H, Choi SH, Kim S, Kim S, Kim DK (2014) Factors associated with caregiver burden in patients with Alzheimer's disease. Psychiatry Investig 11, 152-159.

[7] Torrisi M, Cola MC de, Marra A, Luca R de, Bramanti P, Calabrò RS (2017) Neuropsychiatric symptoms in dementia may predict caregiver burden: a Sicilian exploratory study. Psychogeriatrics 17, 103-107.

[8] Dietzel N, Karrer L, Wolff F, Kratzer A, Hess M, Gräßel E, Kolominsky-Rabas P (2020) Einflussfaktoren auf die Pflegebelastung der Angehörigen von Menschen mit Demenz: der Bayerische Demenz Survey (BayDem). Gesundheitswesen 82, 30-39.

[9] Forte G, Favieri F, Tambelli R, Casagrande M (2020) COVID-19 pandemic in the Italian population: validation of a post-traumatic stress disorder questionnaire and prevalence of PTSD symptomatology. Int J Environ Res Public Health 17, 4151.

[10] Korczyn AD (2020) Dementia in the COVID-19 period. $J$ Alzheimers Dis 75, 1071-1072.

[11] Chew NWS, Lee GKH, Tan BYQ, Jing M, Goh Y, Ngiam NJH, Yeo LLL, Ahmad A, Ahmed Khan F, Napolean Shanmugam G, Sharma AK, Komalkumar RN, Meenakshi PV, Shah K, Patel B, Chan BPL, Sunny S, Chandra B, Ong JJY, Paliwal PR, Wong LYH, Sagayanathan R, Chen JT, Ying $\mathrm{Ng}$ AY, Teoh HL, Tsivgoulis G, Ho CS, Ho RC, Sharma VK (2020) A multinational, multicentre study on the psychological outcomes and associated physical symptoms amongst healthcare workers during COVID-19 outbreak. Brain Behav Immun 88, 559-565.

[12] Boutoleau-Bretonnière $\mathrm{C}$, Pouclet-Courtemanche $\mathrm{H}$, Gillet A, Bernard A, Laure Deruet A, Gouraud I, Mazoue A, Lamy E, Rocher L, Kapogiannis D, El Haj M (2020) The effects of confinement on neuropsychiatric symptoms in Alzheimer's disease during the COVID-19 crisis. J Alzheimers Dis 76, 41-47.

[13] Canevelli M, Valletta M, Toccaceli Blasi M, Remoli G, Sarti G, Nuti F, Sciancalepore F, Ruberti E, Cesari M, Bruno G (2020) Facing dementia during the COVID-19 outbreak. $J$ Am Geriatr Soc 68, 1673-1676.

[14] Lara B, Carnes A, Dakterzada F, Benitez I, PiñolRipoll G (2020) Neuropsychiatric symptoms and quality of life in Spanish patients with Alzheimer's disease 
during the COVID-19 lockdown. Eur J Neurol, doi: 10.1111/ene.14339.

[15] American Psychiatric Association, DSM-5 Task Force (2013) Diagnostic and statistical manual of mental disorders: DSM-5, Fifth edition, American Psychiatric Association, Arlington, VA.

[16] McKhann GM, Knopman DS, Chertkow H, Hyman BT, Jack CR, Kawas CH, Klunk WE, Koroshetz WJ, Manly JJ, Mayeux R, Mohs RC, Morris JC, Rossor MN, Scheltens P, Carrillo MC, Thies B, Weintraub S, Phelps $\mathrm{CH}$ (2011) The diagnosis of dementia due to Alzheimer's disease: recommendations from the National Institute on Aging-Alzheimer's Association workgroups on diagnostic guidelines for Alzheimer's disease. Alzheimers Dement 7, 263-269.

[17] Iadecola C, Duering M, Hachinski V, Joutel A, Pendlebury ST, Schneider JA, Dichgans M (2019) Vascular cognitive impairment and dementia: JACC Scientific Expert Panel. $J$ Am Coll Cardiol 73, 3326-3344.

[18] Olney NT, Spina S, Miller BL (2017) Frontotemporal dementia. Neurol Clin 35, 339-374.

[19] Chui HC, Victoroff JI, Margolin D, Jagust W, Shankle R, Katzman R (1992) Criteria for the diagnosis of ischemic vascular dementia proposed by the State of California Alzheimer's Disease Diagnostic and Treatment Centers. Neurology 42, 473-480.

[20] Custodio N, Montesinos R, Lira D, Herrera-Pérez E, Bardales Y, Valeriano-Lorenzo L (2017) Mixed dementia: A review of the evidence. Dement Neuropsychol 11, 364-370.

[21] Kaufer DI, Cummings JL, Ketchel P, Smith V, MacMillan A, Shelley T, Lopez OL, DeKosky ST (2000) Validation of the NPI-Q, a brief clinical form of the Neuropsychiatric Inventory. J Neuropsychiatry Clin Neurosci 12, 233-239.

[22] Morris GM, Holden TR, Weng H, Xiong C, Coble DW, Cairns NJ, Morris JC (2020) Comparative performance and neuropathologic validation of the AD8 Dementia Screening Instrument. Alzheimer Dis Assoc Disord 34, 112-117.
[23] Bucks RS, Haworth J (2002) Bristol Activities of Daily Living Scale: a critical evaluation. Expert Rev Neurother 2, 669-676.

[24] Johnson SU, Ulvenes PG, Øktedalen T, Hoffart A (2019) Psychometric properties of the General Anxiety Disorder 7Item (GAD-7) Scale in a heterogeneous psychiatric sample. Front Psychol 10, 1713.

[25] Mystakidou K, Tsilika E, Parpa E, Galanos A, Vlahos L (2007) Psychometric properties of the Impact of Event Scale in Greek cancer patients. J Pain Symptom Manage 33, 454461.

[26] Yurdugül H (2008) Minimum sample size for Cronbach's coefficient alpha: A Monte-Carlo study. Hacettepe Üniversitesi Eğitim Fakültesi Dergisi 35, 397-405.

[27] Newman MG, Llera SJ, Erickson TM, Przeworski A, Castonguay LG (2013) Worry and generalized anxiety disorder: a review and theoretical synthesis of evidence on nature, etiology, mechanisms, and treatment. Annu Rev Clin Psychol 9, 275-297.

[28] Doxiades CA (1968) Ekistics: An introduction to the science of human settlements. Hutchinson \& Co. (Publishers) Ltd., London.

[29] Wang H, Li T, Barbarino P, Gauthier S, Brodaty H, Molinuevo JL, Xie H, Sun Y, Yu E, Tang Y, Weidner W, Yu $X$ (2020) Dementia care during COVID-19. Lancet 395, 1190-1191.

[30] Jawaid A (2020) Protecting older adults during social distancing. Science 368, 145.

[31] Monsen KA, Blok J de (2018) Buurtzorg: Nurse-led community care. Creat Nurs 24, 112-117. 